Pacific Journal of Mathematics

ON VALUED, COMPLETE FIELDS AND THEIR
AUTOMORPHISMS 


\title{
ON VALUED, COMPLETE FIELDS AND THEIR AUTOMORPHISMS
}

\author{
HANS ARWED KelleR
}

The following theorem is proved:

THEOREM. Let $K$ be a valued, complete field, and assume that the valuation topology admits countable neighbourhood bases. All the automorphisms of $K$ are continuous if and only if $K$ is not algebraically closed.

Introduction. In this paper we study the following problem: Which valued, complete fields have the property that all their automorphisms are continuous? In the case of Archimedean valuations the answer is well known: There are only two complete fields, $\mathbf{R}$ and $\mathbf{C}$. $\operatorname{Aut}(\mathbf{R})=\{$ Id $\} ; \mathbf{C}$ has exactly two continuous automorphisms, but card $\operatorname{Aut}(\mathbf{C})=2^{\left(2^{\mathrm{x}_{0}}\right)}$, hence very many $\tau \in \operatorname{Aut}(\mathbf{C})$ are discontinuous. Henceforth we shall consider exclusively fields $K$ with non-Archimedean valuations $\phi$ in the sense of Krull. We assume that the valuation topology admits countable neighbourhood bases. Suppose now that $(K, \phi)$ is complete. Our main result is that all the automorphisms of $K$ are continuous if and only if $K$ is not algebraically closed. Thus there is a perfect analogy to the Archimedean case.

If the valuation $\phi$ has rank 1, Hensel's lemma is available and a rather short proof can be given (see $\S 4$ ). The case where $\phi$ has infinite rank requires a new method; our proof in $\$ 5$ will be based on a lemma on solvability of certain infinite systems of equations. The technique of this lemma can be applied to other problems on complete fields; two illustrations for this are given in $\S 6$.

We should like to mention that the paper was motivated by studies in the theory of orthomodular spaces. These are, by definition, vector spaces $E$ endowed with a hermitian form $\Psi$ such that the lattice $L=\{U \subseteq E$ : $\left.U=\left(U^{\perp}\right)^{\perp}\right\}$ of all orthogonally closed subspaces of $(E, \Psi)$ satisfies the orthomodular law: $U \leq V \Rightarrow V=U \vee\left(V \wedge U^{\perp}\right)$ for all $U, V \in L$. Classical examples are the Hilbert spaces over $\mathbf{R}$ or $\mathbf{C}$. In recent years numerous non-classical, infinite dimensional orthomodular spaces have been discovered. All these new spaces are constructed over certain valued, 
complete fields; the valuations in question have infinite rank. Investigations on the particular properties of these spaces $E$ lead, via the fundamental theorem of projective geometry, to maps $f: E \rightarrow E$ which are semilinear, i.e. linear up to some fixed automorphism $\tau$ of the base field $K$. It is then crucial to know that $\tau$ and $\tau^{-1}$ are continuous. We refer to [2] for a comprehensive account on orthomodular spaces.

1. Notations. Let $K$ be a field and $\phi: K \rightarrow \Gamma \cup\{\infty\}$ a Krull valuation on $K$, i.e. $\Gamma$ is an ordered abelian group, written additively, and for all $x, y \in K$ we have (i) $\phi(x)=\infty \Leftrightarrow x=0$, (ii) $\phi(x y)=\phi(x)+$ $\phi(y)$, (iii) $\phi(x+y) \geq \min \{\phi(x), \phi(y)\}$. We assume that $\Gamma \neq\{0\}$ and that $\phi$ is onto. The valuation topology $\mathscr{T}=\mathscr{T}(\phi)$ on $K$ is defined by taking $\left\{U_{\gamma}(a): \gamma \in \Gamma\right\}$, where $U_{\gamma}(a)=\{x \in K: \phi(a-x) \geq \gamma\}$, as a neighbourhood basis of $a \in K$. Notions as "continuity" etc. always refer to this topology. Throughout the paper we make the assumption that $\mathscr{T}(\phi)$ admits a countable zero-neighbourhood basis, or, equivalently, that $\Gamma$ contains a countable cofinal subset. Thus by "valued field" we mean a field with a Krull valuation satisfying this countability condition.

A subgroup $\Delta \leq \Gamma$ is called isolated if $\delta \in \Delta, \gamma \in \Gamma, 0 \leq \gamma \leq \delta$ implies $\gamma \in \Delta$. We set $\mathscr{D}=\mathscr{D}(\Gamma)=\{\Delta \supsetneqq \Gamma: \Delta$ is an isolated subgroup $\}$. Every $\Delta \in \mathscr{D}$ gives rise to a valuation $\Phi_{\Delta}: K \rightarrow(\Gamma / \Delta) \cup\{\infty\}$. The valuation ring corresponding to $\Phi_{\Delta}$ is $A_{\Delta}=\{x \in K: \phi(x) \geq \delta$ for some $\delta \in \Delta\}$. We let $J_{\Delta}$ denote the maximal ideal of $A_{\Delta} ; \hat{K}_{\Delta}=A_{\Delta} / J_{\Delta}$ is the residue field of $A_{\Delta}$. For details see [1, §7].

We denote by $\omega$ the set of all finite ordinals.

\section{The main theorem. Our purpose is to prove}

THEOREM 1. Let the valued field $(K, \phi)$ be complete. Then the following conditions are equivalent:

(a) All automorphisms of $K$ are continuous.

(b) $K$ is not algebraically closed.

The proof will be divided into several steps. We begin with the easy part.

Proof of the implication (a) $\Rightarrow(\mathrm{b})$. Suppose $(K, \phi)$ is complete and algebraically closed. Let $P$ be the prime field. Then $\operatorname{tr} \operatorname{deg}(K \mid P)>\boldsymbol{\aleph}_{0}$. It is easy to construct a transcendence basis $B=\left\{t_{\mu}: \mu \in M\right\}$ for $K$ over $P$ such that $\left\{\phi\left(t_{\mu}\right): \mu \in M\right\}$ has no upper bound in $\Gamma$. Then (by virtue of the general assumption on $\Gamma$ ) there is a sequence $\left(t_{i}\right)_{i \in \omega}, t_{i} \in B$, with 
$\phi\left(t_{i}\right) \rightarrow \infty$ as $i \rightarrow \infty$. Since card $M>\boldsymbol{\aleph}_{0}$ there exists a $\gamma \in \Gamma$ with $\left\{\mu \in M: \phi\left(t_{\mu}\right) \leq \gamma\right\}$ infinite, so we can define a permutation $\sigma: B \rightarrow B$ such that $\phi\left(\sigma\left(t_{i}\right)\right) \leq \gamma$ for all $i \in \omega$. $\sigma$ induces an automorphism $\tau_{0}$ of $P\left(t_{\mu}\right)_{\mu \in M}$ which is not continuous, and $\tau_{0}$ extends to an automorphism $\tau$ : $K \rightarrow K$, since $K$ is algebraically closed. Hence (a) implies (b).

3. On the rank of $\phi$. The set $\mathscr{D}=\mathscr{D}(\Gamma)$ is totally ordered by the inclusion $\subseteq$. The order type of $(\mathscr{D}, \subseteq)$ is called the rank of the valuation $\phi$ and denoted by $\operatorname{rg}(\phi)$. The cofinality of $\mathscr{D}$, denoted by $\operatorname{cf}(\mathscr{D})$, is the least ordinal which is similar to a cofinal subset of $(\mathscr{D}, \subseteq)$. There are two possibilities.

1st case. $\operatorname{cf}(\mathscr{D})=1$. This means that $\mathscr{D}$ has a largest element, say $\Delta_{0}$. Then the valuation $\phi_{\Delta_{0}}: K \rightarrow\left(\Gamma / \Delta_{0}\right) \cup\{\infty\}$ has rank 1 , because $\Delta_{0}$ is maximal. Furthermore, $\phi_{\Delta_{0}}$ defines the same topology on $K$ as $\phi$. Thus, in the proof of Theorem 1, we may replace $\phi$ by $\phi_{\Delta_{0}}$, i.e. we may suppose that $\operatorname{rg}(\phi)=1$.

2nd case. $\operatorname{cf}(\mathscr{D})=\omega$. This means that all $\phi_{\Delta}(\Delta \in \mathscr{D})$ have infinite rank.

We now continue the proof of Theorem 1 . We have to show that the existence of a discontinuous $\tau \in \operatorname{Aut}(K)$ implies that $K$ is algebraically closed. The above two cases must be treated differently.

4. The first case: $\operatorname{rg}(\phi)=1$. We need the following

LEMma 1. Let $(K, \phi)$ be a valued field and suppose that $\tau \in \operatorname{Aut}(K)$ is discontinuous. Then

(i) There is a sequence $\left(v_{i}\right)_{i \in \omega}$ in $K$ such that $v_{i} \rightarrow 0$ and $\tau\left(v_{i}\right) \rightarrow \infty$ as $i \rightarrow \infty$.

(ii) Given $a, b \in K$ and $\gamma \in \Gamma$ there exists $a c \in K$ with $\phi(b-c) \geq \gamma$ and $\phi(a-\tau(c)) \geq \gamma$.

Proof. (i) follows by routine arguments. To show (ii) let $\left(v_{i}\right)_{i \in \omega}$ be as in (i). Put $u_{i}=b+\left(\tau^{-1}(a)-b\right) \cdot v_{i} \cdot\left(1+v_{i}\right)^{-1}$. Then $u_{i} \rightarrow b$ and $\tau\left(u_{i}\right)$ $\rightarrow a$, so we can take $c=u_{j}$ with $j$ sufficiently large.

For the basic properties of henselian fields we refer to [3, Chap. F] or $[1, \S 16]$. 
Lemma 2. Suppose the valued field $(K, \phi)$ is henselian. Let $L$ be the algebraic closure of $K$ and $\psi$ the unique valuation on $L$ which extends $\phi$. If $K$ has a discontinuous automorphism $\tau$, then $K$ is topologically dense in $(L, \psi)$.

Proof. Let $\Gamma$ and $\Gamma^{\prime}$ be the value groups of $\phi$ and $\psi$ respectively. $\Gamma$ is cofinal in $\Gamma^{\prime}$. Let $A=\{x \in K: \phi(x) \geq 0\}$ be the valuation ring of $\phi, \hat{K}$ its residue field and $\hat{:} A \rightarrow \hat{K}$ the canonical epimorphism.

(1) We assert: given $f(x)=\sum_{j=0}^{r} a_{j} X^{j} \in K[X]$ (where $a_{r}=1, r \geq 1$ ) and $\gamma \in \Gamma$ there exist $a_{0}^{\prime}, \ldots, a_{r}^{\prime} \in K$ such that

(i) $\phi\left(a_{j}^{\prime}-a_{j}\right) \geq \gamma$ for $0 \leq j \leq r-1, a_{r}^{\prime}=1$.

(ii) $f^{*}(X)=\sum_{j=0}^{r} a_{j}^{\prime} X^{j}$ decomposes into linear factors in $K[X]$.

We show this by induction on $r$. The case $r=1$ is trivial, so let $r \geq 2$. Using Lemma 1, (ii) we find $c_{0}, \ldots, c_{r-1} \in K$ such that $\phi\left(a_{j}-\tau\left(c_{j}\right)\right) \geq \gamma$ for $0 \leq j \leq r-1$, and $\phi\left(1-c_{1}\right)>0, \phi\left(0-c_{j}\right)>0$ for $j=0,2, \ldots, r-$ 1. Let $h(X)=\sum_{j=0}^{r} c_{j} X^{j}$ where $c_{r}=1$. Then $h(X) \in A[X], h(X)$ is monic, and $\hat{h}(X)=\sum_{j=0}^{r} \hat{c}_{j} X^{j}=X+X^{r} \in \hat{K}[X]$ has a simple root in $\hat{K}$. It follows that $h(X)$ has a root in $K$, hence also $h^{\tau}(X)=\sum_{j=0}^{r} \tau\left(c_{j}\right) X^{j}$ has a root in $K$. Thus $h^{\tau}(X)=(X-t) g(X)$ where $t \in K$ and $g(X)=$ $\sum_{j=0}^{r-1} d_{j} X^{j} \in K[X], d_{r-1}=1$. By the induction hypothesis, applied to $g(X)$ and $\varepsilon=\max \{\gamma, \gamma-\phi(t)\}$, there are $d_{0}^{\prime}, \ldots, d_{r-1}^{\prime} \in K$ with $\phi\left(d_{j}^{\prime}-d_{j}\right) \geq \varepsilon$ for $0 \leq j \leq r-2, d_{r-1}^{\prime}=1$, such that

$$
g^{*}(X)=\sum_{j=0}^{r-1} d_{j}^{\prime} X^{j}
$$

is a product of linear factors in $K[X]$. We determine $a_{0}^{\prime}, \ldots, a_{r}^{\prime}$ so that $\sum_{j=0}^{r} a_{j}^{\prime} X^{j}=(X-t) g^{*}(X)$. Clearly (ii) holds, and (i) is readily checked.

(2) Now let $w \in L$ and $\delta \in \Gamma^{\prime}$ be given. Let

$$
f(X)=\operatorname{irr}(w, K)=\sum_{j=0}^{r} a_{j} X^{j} \in K[X]
$$

and choose $\gamma \in \Gamma$ such that $\gamma \geq \max \{r \cdot \delta-j \cdot \psi(w): 0 \leq j \leq r-1\}$. By (1) there are $a_{0}^{\prime}, \ldots, a_{r}^{\prime} \in K$ with $\phi\left(a_{j}^{\prime}-a_{j}\right) \geq \gamma$ for $0 \leq j \leq r-1$, $a_{r}^{\prime}=1$, such that

$$
f^{*}(X)=\sum_{j=0}^{r} a_{j}^{\prime} X^{j}=\prod_{k=1}^{r}\left(X-t_{k}\right)
$$

where $t_{1}, \ldots, t_{r} \in K$. Then

$$
\prod_{k=1}^{r}\left(w-t_{k}\right)=f^{*}(w)=f^{*}(w)-f(w)=\sum_{j=0}^{r-1}\left(a_{j}^{\prime}-a_{j}\right) w^{j} .
$$


It follows that

$$
\begin{aligned}
\sum_{k=1}^{r} \psi\left(w-t_{k}\right) & \geq \min \left\{\psi\left(\left(a_{j}^{\prime}-a_{j}\right) w^{j}\right): 0 \leq j \leq r-1\right\} \\
& \geq \min \{\gamma+j \cdot \psi(w): 0 \leq j \leq r-1\} \geq r \cdot \delta .
\end{aligned}
$$

Hence for some $k \in\{1, \ldots, r\}$ we have $\psi\left(w-t_{k}\right) \geq \delta$ and $t_{k} \in K$. Hence $K$ is dense in $L$.

Corollary 1. Let $(K, \phi)$ be a complete field, $\operatorname{rg}(\phi)=1$. If $K$ admits a discontinuous automorphism, then $K$ is algebraically closed.

Proof. $(K, \phi)$ is a henselian field ([3], p. 198) and the assertion follows immediately from the lemma, because $K$ is topologically closed in $L$.

Thus we have the implication (b) $\Rightarrow$ (a) in Theorem 1 in case $\operatorname{rg}(\phi)=$ 1.

COROLlaRY 2. Let $(K, \phi)$ be a henselian field which has a discontinuous automorphism. Then $K$ is separably algebraically closed. Furthermore, the value group $\Gamma$ of $\phi$ is divisible and the residue field $\hat{K}_{\Delta}$ is algebraically closed for all $\Delta \in \mathscr{D}$.

Proof. With the notations of Lemma 2, let $K_{s}=\{w \in L: w$ is separable over $K\}, \psi^{\prime}=\left.\psi\right|_{K_{s}}$, and let $G=\operatorname{Gal}\left(K_{s} \mid K\right)$. Since $\psi^{\prime}$ is the unique extension of $\phi$ to $K_{s}, \psi^{\prime} \circ \sigma=\psi^{\prime}$ for every $\sigma \in G$. This implies that every $\sigma \in G$ is continuous. $K$ is the fixed field of $G$, consequently $K=\bigcap_{\sigma \in G} \operatorname{Ker}\left(\sigma-\operatorname{Id}_{K_{s}}\right)$ is topologically closed in $K_{s}$. By Lemma 2, $K$ is dense in $L$, so dense in $K_{s}$. We conclude that $K=K_{s}$. The last two statements follow from the fact that $(L, \psi)$ is an immediate extension of $(K, \phi)$, because $K$ is dense in $L$.

5. The second case: $\operatorname{cf}(\mathscr{D})=\omega$.

LEMMA 3. Suppose the valued field $(K, \phi)$ is complete and $\operatorname{cf}(\mathscr{D})=\omega$. Let $\left(v_{i}\right)_{i \in \omega}$ be a sequence in $K$ with $v_{i} \rightarrow 0$ as $i \rightarrow \infty$. Let $q(X, Z)=$ $\sum_{k, l} a_{k l} X^{k} Z^{l}$ be a polynomial in $K[X, Z]$ with $q(0,0)=0$. Then there exist elements $c_{0}, c_{1}, \ldots, c_{m}, \ldots$ in $K$ such that

$$
c_{m}=q\left(c_{m+1}, v_{m}\right)=\sum_{k, l} a_{k l} c_{m+1}^{k} v_{m}^{l} \quad \text { for all } m \in \omega .
$$


Proof. Let $S$ be the subring of $K$ generated by the coefficients $a_{k l}$ of $q(X, Z)$. We note that $\{\phi(s): s \in S\}$ has a lower bound in $\Gamma$, by virtue of the hypothesis $\operatorname{cf}(\mathscr{D})=\omega$. Let $Y_{i}(i \in \omega)$ be new variables and form the polynomial ring $R=S\left[Y_{i}\right]_{i \in \omega}$. For each $n \in \omega$ we define recursively $f_{n}^{(0)}, \ldots, f_{n}^{(n)} \in R$ by

$$
\left\{\begin{array}{l}
f_{n}^{(0)}=q\left(0, Y_{n}\right) \\
f_{n}^{(j)}=q\left(f_{n}^{(j-1)}, Y_{n-j}\right) \quad \text { for } j=1, \ldots, n .
\end{array}\right.
$$

Thus $f_{n}^{(j)}$ is a polynomial in $Y_{n}, Y_{n-1}, \ldots, Y_{n-j}$. We put $g^{(0)}=f_{0}^{(0)}, g^{(n)}=$ $f_{n}^{(n)}-f_{n-1}^{(n-1)}$ for $n \geq 1$; then

$$
f_{n}^{(n)}=\sum_{i=0}^{n} g^{(i)} \quad \text { for all } n \in \omega .
$$

We claim that every $g^{(n)} \in R$ has the form

$$
g^{(n)}=Y_{n} \cdot h^{(n)}, \quad \text { where } h^{(n)} \in R .
$$

In fact, for $n=0$ this is true because $q(0,0)=0$. Let $n \geq 1$. Substituting $Y_{n}=0$ in (2) we see by induction on $j$ that $\left.f_{n}^{(j)}\right|_{Y_{n}=0}=f_{n-1}^{(j-1)}$ for $j=1, \ldots, n$. For $j=n$ this means that $g^{(n)}$ vanishes at $Y_{n}=0$, as claimed.

We define the homomorphism $\sigma: R \rightarrow R$ by $\left.\sigma\right|_{S}=\mathrm{Id}_{S}, \sigma\left(Y_{i}\right)=Y_{i+1}$ $\left(i \in \omega\right.$ ). From (2) it follows easily that $\sigma\left(f_{n}^{(j)}\right)=f_{n+1}^{(j)}$ for $j=0, \ldots, n$, in particular

$$
\sigma\left(f_{n}^{(n)}\right)=f_{n+1}^{(n)} \quad \text { for all } n \in \omega .
$$

For $n, m \in \omega$ we set $g_{m}^{(n)}=\sigma^{m}\left(g^{(n)}\right)$, where $\sigma^{0}=\operatorname{Id}_{R}$. By (4) we have

$$
g_{m}^{(n)}=Y_{n+m} \cdot h_{m}^{(n)}, \quad \text { where } h_{m}^{(n)}=\sigma^{m}\left(h^{(n)}\right) \in R .
$$

Now we consider the homomorphism $\eta: R \rightarrow K$ defined by $\left.\eta\right|_{S}=\operatorname{Id}_{S}$, $\eta\left(Y_{\imath}\right)=v_{i}(i \in \omega)$. We put $d_{m}^{(n)}=\eta\left(g_{m}^{(n)}\right)$; then, by (6),

$$
d_{m}^{(n)}=v_{n+m} \cdot \eta\left(h_{m}^{(n)}\right) \text {. }
$$

We claim that for all $m \in \omega$ we have

$$
d_{m}^{(n)} \rightarrow 0 \quad \text { as } n \rightarrow \infty .
$$

In fact, $\phi$ is bounded from below on $S$ and on $\left\{v_{i}: i \in \omega\right\}$, therefore (since $\operatorname{cf}(\mathscr{D})=\omega$ ) also on $\eta(R)=$ the subring generated by $S$ and the $v_{\imath}$ 's. Now $v_{n+m} \rightarrow 0$ as $n \rightarrow \infty$, so (8) follows from (7). 
By (8) and by completeness, the series $\sum_{i=0}^{\infty} d_{m}^{(i)}$ converges. We set, for $m \in \omega, c_{m}=\sum_{i=0}^{\infty} d_{m}^{(i)}$ and verify that these elements satisfy (1). Let $m \in \omega$. For any $n \in \omega$ we have

$$
\begin{aligned}
\sum_{i=0}^{n+1} g^{(i)} & =f_{n+1}^{(n+1)}=q\left(f_{n+1}^{(n)}, Y_{0}\right) \quad \text { by }(2),(3) \\
& =q\left(\sigma\left(f_{n}^{(n)}\right), Y_{0}\right) \quad \text { by }(5) \\
& =q\left(\sum_{i=0}^{n} \sigma\left(g^{(i)}\right), Y_{0}\right) \quad \text { by }(3) .
\end{aligned}
$$

Applying $\sigma^{m}$ we get

$$
\sum_{i=0}^{n+1} g_{m}^{(i)}=q\left(\sum_{i=0}^{n} g_{m+1}^{(i)}, Y_{m}\right)
$$

and applying $\eta$ we get

$$
\sum_{i=0}^{n+1} d_{m}^{(i)}=q\left(\sum_{i=0}^{n} d_{m+1}^{(i)}, v_{m}\right)
$$

Now we let $n \rightarrow \infty$ and obtain (1). The proof is complete.

We now show how Lemma 3 can be used to derive consequences from the existence of a discontinuous $\tau \in \operatorname{Aut}(K)$.

LEMMA 4. Let $(K, \phi)$ be complete, $\operatorname{cf}(\mathscr{D})=\omega$. If $K$ admits a discontinuous automorphism $\tau$, then every residue field $\hat{K}_{\Delta}(\Delta \in \mathscr{D})$ is algebraically closed.

Proof. Fix $\Delta \in \mathscr{D}$ and let $\hat{:} A_{\Delta} \rightarrow \hat{K}_{\Delta}$ be the canonical map. Let $\hat{p}(X)=\hat{a}_{0}+\hat{a}_{1} X+\cdots+\hat{a}_{r-1} X^{r-1}+X^{r} \in \hat{K}_{\Delta}[X]$; we must find a root of $\hat{p}(X)$ in $\hat{K}_{\Delta}$. By Lemma 1 , (i) there is a sequence $\left(v_{i}\right)_{i \in \omega}$ in $K$ with $v_{i} \rightarrow 0$ and $\tau\left(v_{i}\right) \rightarrow \infty$ as $i \rightarrow \infty$. We apply Lemma 3 to this sequence $\left(v_{i}\right)$ and the polynomial

$$
\begin{aligned}
q(X, Z)= & \tau^{-1}\left(a_{0}\right) Z^{r}+\tau^{-1}\left(a_{1}\right) X Z^{r-1} \\
& +\cdots+\tau^{-1}\left(a_{r-1}\right) X^{r-1} Z+X^{r} .
\end{aligned}
$$

We get elements $c_{m} \in K(m \in \omega)$ such that $c_{m}=q\left(c_{m+1}, v_{m}\right)$ for all $m$. We put $d_{m}=\tau\left(c_{m}\right), w_{m}=\tau\left(v_{m}\right)$; then $d_{m}=q^{\tau}\left(d_{m+1}, w_{m}\right)$, that is

$$
d_{m}=a_{0} w_{m}^{r}+a_{1} d_{m+1} w_{m}^{r-1}+\cdots+a_{r-1} d_{m+1}^{r-1} w_{m}+d_{m+1}^{r}
$$

for all $m \in \omega$. Choose a $\Delta^{\prime} \in \mathscr{D}$ such that $\Delta \leq \Delta^{\prime}$ and $d_{0}, w_{0} \in A_{\Delta^{\prime}}$; this is possible since $\operatorname{cf}(\mathscr{D})=\omega$. Note that $a_{0}, \ldots, a_{r-1} \in A_{\Delta^{\prime}}$. Now $w_{i} \rightarrow \infty$ as $i \rightarrow \infty$, hence we have $w_{0}, \ldots, w_{n-1} \in A_{\Delta^{\prime}}, w_{n} \notin A_{\Delta^{\prime}}$ for some $n \in \omega$. 
We claim that $d_{0}, \ldots, d_{n} \in A_{\Delta^{\prime}}$. In fact, $d_{0} \in A_{\Delta^{\prime}}$ by the choice of $\Delta^{\prime}$, and if $d_{j} \in A_{\Delta^{\prime}}, j<n$, then equation (9) with $m=j$ shows that $d_{j+1}$ is integral over $A_{\Delta^{\prime}}$, hence $d_{j+1} \in A_{\Delta^{\prime}}$ because $A_{\Delta^{\prime}}$ is integrally closed.

Now consider equation (9) with $m=n$. We divide it by $w_{n}^{r}$ and get

$$
\frac{d_{n}}{w_{n}^{r}}=a_{0}+a_{1} t+\cdots+a_{r-1} t^{r-1}+t^{r}, \quad \text { where } t=\frac{d_{n+1}}{w_{n}} .
$$

We have $d_{n} \in A_{\Delta^{\prime}}$ and $w_{n} \notin A_{\Delta^{\prime}}$, so $d_{n} / w_{n}^{r} \in J_{\Delta^{\prime}} \subseteq J_{\Delta} \subseteq A_{\Delta}$. It follows that $t \in A_{\Delta}$, and reducing (10) modulo $J_{\Delta}$ we get $0=\hat{a}_{0}+\cdots+\hat{a}_{r-1} \hat{t}^{r-1}+\hat{t}^{r}$, i.e. $\hat{t} \in \hat{K}_{\Delta}$ is a root of $\hat{p}(X)$.

LEMMA 5. Suppose $(K, \phi)$ is complete, $\operatorname{cf}(\mathscr{D})=\omega$. If every residue field $\hat{K}_{\Delta}(\Delta \in \mathscr{D})$ is algebraically closed, then $K$ is algebraically closed.

Proof. We fix in $\mathscr{D}$ a chain $\Delta_{0} \leq \Delta_{1} \leq \cdots \leq \Delta_{n} \leq \cdots$ with $\bigcup_{n=0}^{\infty} \Delta_{n}$ $=\Gamma$. We write $A_{n}, J_{n}, \hat{K}_{n}$ instead of $A_{\Delta_{n}}, J_{\Delta_{n}}, \hat{K}_{\Delta_{n}}$, and we let $\pi_{n}: A_{n} \rightarrow \hat{K}_{n}$ be the canonical map. Let be given $p(X)=\sum_{j=0}^{r} a_{j} X^{j} \in K[X]$. We may assume that $a_{0}, \ldots, a_{r} \in A_{0}, a_{r}=1$. Put

$$
\hat{p}_{n}(X)=\sum_{j=0}^{r} \pi_{n}\left(a_{j}\right) X^{j} \in \hat{K}_{n}[X] .
$$

We construct recursively a sequence $\left(t_{n}\right)_{n \in \omega}$ in $K$ with

(i) $t_{n} \in A_{0} \subseteq A_{n}$, and $\pi_{n}\left(t_{n}\right) \in \hat{K}_{n}$ is a root of $\hat{p}_{n}(X)$ (for all $n \in \omega$ ).

(ii) $\pi_{n-1}\left(t_{n-1}\right)=\pi_{n-1}\left(t_{n}\right)$ for all $n \geq 1$.

Suppose $t_{0}, \ldots, t_{n-1}$ have already been constructed satisfying (i), (ii). Since $\hat{K}_{n}$ is algebraically closed, there are $w_{1}, \ldots, w_{r} \in A_{n}$ such that $\hat{p}_{n}(X)=\prod_{k=1}^{r}\left(X-\pi_{n}\left(w_{k}\right)\right)$. Then $p\left(w_{k}\right) \in J_{n} \subseteq J_{0} \subseteq A_{0}$, i.e. $a_{0}$ $+\cdots+a_{r-1} w_{k}^{r-1}+w_{k}^{r} \in A_{0}$, which implies that $w_{k} \in A_{0}$ for all $k=$ $1, \ldots, r$. The coefficients of $p(X)-\prod_{k=1}^{r}\left(X-w_{k}\right)$ lie in $J_{n}$, hence in $J_{n-1}$, consequently $\hat{p}_{n-1}(X)=\prod_{k=1}^{r}\left(X-\pi_{n-1}\left(w_{k}\right)\right)$. Therefore $\pi_{n-1}\left(t_{n-1}\right)$ must be equal to $\pi_{n-1}\left(w_{k}\right)$ for some $k \in\{1, \ldots, r\}$. We put $t_{n}=w_{k}$; clearly (i) and (ii) are satisfied.

Now by (ii) we have $t_{n}-t_{n-1} \in J_{n-1}$ for $n \geq 1$, which shows that $\left(t_{n}\right)_{n \in \omega}$ is a Cauchy sequence. It follows from (i) that $t=\lim _{n \rightarrow \infty} t_{n} \in K$ is a root of $p(X)$. This proves the lemma.

Combining Lemmas 4 and 5 we get the implication $(b) \Rightarrow(a)$ in Theorem 1 in the case where $\operatorname{cf}(\mathscr{D})=\omega$. The proof of Theorem 1 is complete.

6. Applications. We give two applications of the technique of $\S 5$. Theorem 2 below shows that all valued, complete fields have some purely algebraic features in common. 
THEOREM 2. Let $(K, \phi)$ be a valued, complete field and $F$ a proper subfield of $K$. Then $K$ is not a purely transcendental extension of $F$.

Proof. Suppose, indirectly, that $K$ were purely transcendental over $F$. It is sufficient to consider the case where $\operatorname{tr} \operatorname{deg}(K \mid F)=1$, thus $K=F(t)$ with $t$ transcendental over $F$. Of course $K$ is not algebraically closed. Again (cf. §3) we may assume that either $\operatorname{rg}(\phi)=1$ or $\operatorname{cf}(\mathscr{D})=\omega$.

1. Suppose $\operatorname{rg}(\phi)=1$. Replacing $t$ by $c \cdot t$ or $c \cdot t^{-1}(0 \neq c \in F)$ if necessary we may assume that $\phi(t)>0$. Define $\tau \in \operatorname{Aut}(K)$ by $\left.\tau\right|_{F}=\operatorname{Id}_{F}$, $\tau(t)=t^{-1}$. Then $t^{i} \rightarrow 0$ as $i \rightarrow \infty$, but $\tau\left(t^{i}\right)=t^{-i} \nrightarrow 0$, which contradicts Theorem 1.

2. Suppose $\operatorname{cf}(\mathscr{D})=\omega$. It is clear that $\{\phi(v): 0 \neq v \in F\} \subseteq \Gamma$ has no upper bound in $\Gamma$, for otherwise there would exist a $\Delta \in \mathscr{D}$ with $\phi(t) \in \Delta$ and $\phi(v) \in \Delta$ for all $0 \neq v \in F$, so $\phi(x) \in \Delta$ for all $0 \neq x \in F(t)=K$, which is impossible. It follows that there is a sequence $\left(v_{i}\right)_{i \in \omega}, 0 \neq v_{i} \in F$, with $v_{i} \rightarrow 0$ as $i \rightarrow \infty$. Let $q(X, Z)=X^{2}+t \cdot Z \in K[X, Z]$. We use Lemma 3 to find elements $c_{m} \in K(m \in \omega)$ such that $c_{m}=q\left(c_{m+1}, v_{m}\right)$ for all $m \in \omega$. Write $c_{m}=f_{m}(t) / g_{m}(t)$, where $f_{m}(t), g_{m}(t) \in F[t]$, relatively prime. We get

$$
\frac{f_{m}(t)-v_{m} \cdot t \cdot g_{m}(t)}{g_{m}(t)}=\frac{f_{m+1}^{2}(t)}{g_{m+1}^{2}(t)} \text { for all } m \text {. }
$$

By unique factorization in $F[t]$ we conclude that $g_{m}(t)=g_{m+1}^{2}(t)$ for all $m$, therefore $g_{m}(t)=1$. We are left with

$$
f_{m}(t)-v_{m} \cdot t=f_{m+1}^{2}(t) \text { for all } m \in \omega .
$$

Since $0 \neq v_{m} \in F$ we must have $\operatorname{deg} f_{m} \neq 0$ for all $m$, but then $\operatorname{deg} f_{m}=$ $2 \cdot \operatorname{deg} f_{m+1}>\operatorname{deg} f_{m+1}$ for all $m$, a contradiction. This proves the theorem.

Our second application is suggested by the theory of orthomodular spaces. As mentioned in the introduction, all known non-classical examples of such spaces are constructed over certain valued, complete fields. One may ask if some of these ground fields can be embedded algebraically into $\mathbf{R}$; such an embedding would yield a quick answer to several open problems. However, we now show

THEOREM 3. A (non-Archimedeanly) valued, complete field $(K, \phi)$ cannot be algebraically isomorphic to a subfield of the reals $\mathbf{R}$.

Proof. Suppose there were a monomorphism $\rho: K \rightarrow \mathbf{R}$. We first consider the case where $\operatorname{cf}(\mathscr{D})=\omega$. 
(1) We construct a sequence $\left(v_{i}\right)_{i \in \omega}$ in $K$ with

$$
v_{i} \rightarrow 0 \text { as } i \rightarrow \infty \text {, and } \rho\left(v_{i}\right)>1 \text { for all } i \in \omega \text {. }
$$

We choose $\Delta_{i} \in \mathscr{D}(i \in \omega)$ such that $\Delta_{0} \supsetneqq \cdots \lesseqgtr \Delta_{1} \lesseqgtr \ldots, \bigcup_{i=0}^{\infty} \Delta_{i}$ $=\Gamma$. For every $i \in \omega$ we pick a $b_{i} \in A_{\Delta_{i+1}} \backslash A_{\Delta_{1}}$ with $\rho\left(b_{i}\right)>0$, then we choose integers $r_{i} \in \mathbf{Z}$ so that $\rho\left(b_{i}\right)^{r_{i}}>\rho\left(b_{i+1}\right)$. Put $v_{i}=b_{i}^{r_{i}} / b_{i+1}$. Clearly $\rho\left(v_{i}\right)>1$, and by choice of the $b_{i}$ 's we have $v_{i} \in J_{\Delta_{t+1}}$, hence $v_{i} \rightarrow 0$.

(2) Let $q(X, Z)=X^{2}+Z$. We use Lemma 3 to obtain elements $c_{m} \in K(m \in \omega)$ such that $c_{m}=q\left(c_{m+1}, v_{m}\right)$ for all $m$. Put $d_{m}=\rho\left(c_{m}\right)$, $w_{m}=\rho\left(v_{m}\right) ;$ then

$$
d_{m}=d_{m+1}^{2}+w_{m} \quad \text { for all } m \in \omega .
$$

But $d_{m}, w_{m} \in \mathbf{R}$ and $w_{m}>1$ by construction, so it is obvious that (11) leads to a contradiction.

It remains to consider the case where $\operatorname{rg}(\phi)=1$. We shall prove, more generally, that no henselian field can be embedded into $\mathbf{R}$. In fact, let $(K, \phi)$ be henselian. Let $A$ be the valuation ring of $\phi, J$ its maximal ideal and $\hat{K}=A / J$. If char $\hat{K} \neq 2$, then $1+J \subseteq K^{2}$, hence $\rho(1+J) \subseteq \mathbf{R}^{2}$. But this is impossible, because $\rho(1+J)=1+\rho(J)$ is a coset of a non-zero additive subgroup of $\mathbf{R}$ and therefore it must contain negative numbers. Now suppose char $\hat{K}=2$. Take any $a \in J, a \neq 0$. Then $f(X)=X^{2}-X$ $-n \cdot a$ has a root in $K$ for every $n \in \mathbf{Z}$. Choose $n \in \mathbf{Z}$ such that $n \cdot \rho(a)<-1 / 4$; then $f^{\rho}(X)=X^{2}-X-n \cdot \rho(a)$ is irreducible over $\mathbf{R}$, a contradiction. This completes the proof.

\section{REFERENCES}

[1] O. Endler, Valuation Theory, Springer, Berlin, 1972.

[2] H. Gross and U. M. Künzi, On a class of orthomodular quadratic spaces, to appear in Enseign. Math.

[3] P. Ribenboim, Théorie des Valuations, Les Presses de I'Université de Montreal, 1964.

Received July 9, 1984. The author was partially supported by DIUC (No. 30/83) and by FNC (No. 0649).

\section{P. Universidad Católica \\ CASILla 6177}

CAMPUS SAN JoAquín

Santiago de Chile 


\title{
PACIFIC JOURNAL OF MATHEMATICS EDITORS
}

V. S. VARADARAJAN (Managing Editor)
University of California
Los Angeles, CA 90024
HEBERT ClEMENS
University of Utah
Salt Lake City, UT 84112
CHARLES R. DEPRIMA
California Institute of Technology
Pasadena, CA 91125

R. FINN

Stanford University

Stanford, CA 94305

HERMANN FLASCHKA

University of Arizona

Tucson, AZ 85721

Ramesh A. Gangolli

University of Washington

Seattle, WA 98195

ROBION KIRBY

University of California

Berkeley, CA 94720
C. C. MOORE

University of California

Berkeley, CA 94720

H. SAMELSON

Stanford University

Stanford, CA 94305

HaRold Stark

University of California, San Diego

La Jolla, CA 92093

\section{ASSOCIATE EDITORS}

\author{
R. ARENS \\ E. F. BECKENBACH \\ B. H. NeumanN \\ F. Wolf \\ K. YoSHIDA \\ (1906-1982)
}

\section{SUPPORTING INSTITUTIONS}

UNIVERSITY OF ARIZONA
UNIVERSITY OF BRITISH COLUMBIA
CALIFORNIA INSTITUTE OF TECHNOLOGY
UNIVERSITY OF CALIFORNIA
MONTANA STATE UNIVERSITY
UNIVERSITY OF NEVADA, RENO
NEW MEXICO STATE UNIVERSITY
OREGON STATE UNIVERSITY

UNIVERSITY OF OREGON

UNIVERSITY OF SOUTHERN CALIFORNIA

STANFORD UNIVERSITY

UNIVERSITY OF HAWAII

UNIVERSITY OF TOKYO

UNIVERSITY OF UTAH

WASHINGTON STATE UNIVERSITY UNIVERSITY OF WASHINGTON

The Supporting Institutions listed above contribute to the cost of publication of this Journal, but they are not owners or publishers and have no responsibility for its content or policies.

Mathematical papers intended for publication in the Pacific Journal of Mathematics should be in typed form or offset-reproduced (not dittoed), double spaced with large margins. Please do not use built up fractions in the text of the manuscript. However, you may use them in the displayed equations. Underline Greek letters in red, German in green, and script in blue. The first paragraph must be capable of being used separately as a synopsis of the entire paper. In particular it should contain no bibliographic references. Please propose a heading for the odd numbered pages of less than 35 characters. Manuscripts, in triplicate, may be sent to any one of the editors. Please classify according to the scheme of Math. Reviews, Index to Vol. 39. Supply name and address of author to whom proofs should be sent. All other communications should be addressed to the managing editor, or Elaine Barth, University of California, Los Angeles, California 90024.

There are page-charges associated with articles appearing in the Pacific Journal of Mathematics. These charges are expected to be paid by the author's University, Government Agency or Company. If the author or authors do not have access to such Institutional support these charges are waived. Single authors will receive 50 free reprints; joint authors will receive a total of 100 free reprints. Additional copies may be obtained at cost in multiples of 50 .

The Pacific Journal of Mathematics is issued monthly as of January 1966. Regular subscription rate: $\$ 190.00$ a year (5 Vols., 10 issues). Special rate: $\$ 95.00$ a year to individual members of supporting institutions.

Subscriptions, orders for numbers issued in the last three calendar years, and changes of address should be sent to Pacific Journal of Mathematics, P.O. Box 969, Carmel Valley, CA 93924, U.S.A. Old back numbers obtainable from Kraus Periodicals Co., Route 100, Millwood, NY 10546.

The Pacific Journal of Mathematics at P.O. Box 969, Carmel Valley, CA 93924 (ISSN 0030-8730) publishes 5 volumes per year. Application to mail at Second-class postage rates is pending at Carmel Valley, California, and additional mailing offices. Postmaster: Send address changes to Pacific Journal of Mathematics, P.O. Box 969, Carmel Valley, CA 93924.

\section{PUBLISHED BY PACIFIC JOURNAL OF MATHEMATICS, A NON-PROFIT CORPORATION}

Copyright $\subset 1986$ by Pacific Journal of Mathematics 


\section{Pacific Journal of Mathematics}

\section{Vol. 121, No. 2 December, 1986}

Jorge Almeida, Minimal nonpermutative pseudovarieties of semigroups.

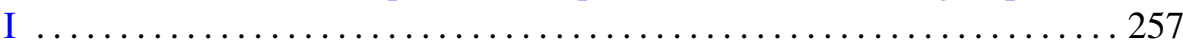

Jorge Almeida, Minimal nonpermutative pseudovarieties of semigroups.

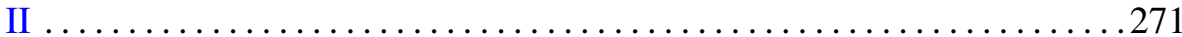

Carlos Andradas Heranz and José Manuel Gamboa Mutuberría, On

projections of real algebraic varieties $\ldots \ldots \ldots \ldots \ldots \ldots \ldots \ldots \ldots 281$

Zeev Ditzian, Inverse theorems for multidimensional Bernstein operators . . 293

M. Furi and Maria Patrizia Pera, A continuation principle for forced oscillations on differentiable manifolds $\ldots \ldots \ldots \ldots \ldots \ldots \ldots \ldots \ldots . \ldots . \ldots . \ldots 321$

James J. Hebda, The collars of a Riemannian manifold and stable

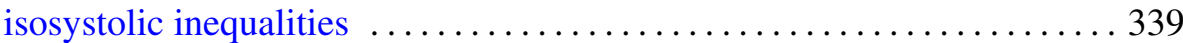

Henryk Hecht and Dragan Miličić, Character identities and asymptotic behavior of matrix coefficients of discrete series ................ 357

Piotr Jakóbczak, The boundary regularity of the solution of the $\bar{\partial}$-equation in the product of strictly pseudoconvex domains $\ldots \ldots \ldots \ldots \ldots \ldots . \ldots 371$

Krzysztof Jarosz, Isometries between injective tensor products of Banach

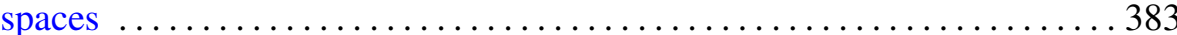

Hans Keller, On valued, complete fields and their automorphisms ........ 397

David Masser and Peter Man-Kit Shiu, On sparsely totient numbers . . . . 407

Tze-Beng Ng, Vector bundles over $(8 k+3)$-dimensional manifolds

Thomas Joseph Ransford, The spectrum of an interpolated operator and analytic multivalued functions

Akihito Uchiyama, On the radial maximal function of distributions 467 Jang-Mei Gloria Wu, On singularity of harmonic measure in space 